\title{
A Relation Merging Technique For Relational Databases*
}

\author{
Victor M. Markowitz ${ }^{\dagger}$ \\ Computer Science and Research \\ Information and Computing Sciences Division \\ Lawrence Berkeley Laboratory \\ 1 Cyclotron Road \\ Berkeley, CA 94720
}

February 1992

Published in the Proceedings of the Eighth International Conference on Data Engineering, 1992

\footnotetext{
* This work was supported by the Applied Mathematical Sciences Research Program and the Office of Health and Environmental Reseanch Program, of the Oftice of Energy Research. U.S. Department of Energy, under Contract DE-ACO3$76 \mathrm{SFO00} 98$.

${ }^{\dagger}$ Author's E-mail address: V.Markowitz@lbl.gov Office phone number:(\$10)486-683 


\title{
A Relation Merging Techiviqe for Relational databases*
}

\author{
Victor M. Markowitz. \\ Computer Science Research and Development \\ Information and Computing Sciences Division. \\ Lawrence Berkeley Laboratory \\ 1 Cyclotron Road, Berkeley, CA 94720
}

\begin{abstract}
Relation merging is employed in relational databases in order to reduce the need for joining relations. Merging, however, can create unnormalized relations. In this paper we propose a merging technique that preserves the high (Boyce-Codd) normal form of relational schemas consisting of relation-schemes, key dependencies, referential integrity constraints, and null constraints. The additional constraints generated by this merging technique can be effectively maintained using the mechanisms provided by several relational database managemunt systems.
\end{abstract}

\section{Introduction}

Relational schema design usually pursues the development of normalized schentas. Normalization leads to decreased data reduridancy and therefore implies simpler procedures for maintaining database consistency and better update performance. The nomalization process tends to increase the number of relations by splitting unnormalized relations into smaller, normalized, relations. Conversely, decreasing the number of relations in a database by merging relations reduces the need for joining relations, and usually results in a better access performance. The process of merging relations, however, may conflice with normalization by creating unnormalized relations. Ideally, the design process should result in a relational schema which is both nor" malized and has as few as possible relation-schemes. This goal is pursued by both normalization (e.g. see [1]) and Entity-Relationship oriented methodologies for designing relational schermas (e.g. see [14]). We examine briefly the shortcomings of the merging techniques involved in these methodologies.

Relation merging was first used in synthesis normalization algorithms. Thus, the synthesis normalization

\footnotetext{
* Issued as technical report LBL-27842. This work was supported by the Applied Mathermatical Sciences Research Program and the Office of Health and Environmental Resench Program, of the Office of Energy Research, U.S. Departmeat of Energy, under Contract DE-ACO376 SFOO098.
}

algorithm presented in [1] involves a step of merging relations with equivalent keys. Consider, for example, two relation-schemes, TEACH (COURSE, FACULTY) and OFFER (COURSE, DEPARTMENT), both having COURSE as key. Following the synthesis algorithm of [1], these relationschemes can be merged into a new relation-scheme whose key is also COURSE: ASSIGN (COURSE, FACULTY, DEPART. MENT). Suppose that the attributes of TEACH and OFFER are not allowed to have null values. Then ASSIGN has equivalent information-capacity [8] with TEACH and OFFER, only if attributes FACULTY and DEPARTMENT are allowed to have null values in ASSIGN, such that in every A.SSIGN tuple at least one of these attributes has a non-null value. Such restrictions, defining the way in which nulls should apnear in relations, were disregarded in the early normalization algorithms. These restrictions can be expli. citly expressed using null constraints [9], or implicitly expressed using the Universal Relation assumptions [10].

An altemative approach to relational schema design has been proposed by the proponents of data models that have more semantic intuition, such as the EntityRelationship (ER) [2] and Extended Entity-Relationship (EER) ([11], [14]) models. The ER and EER models are widely used for designing relational schemas: first, an ER. or EER schema is specified, and then the ER or EER schema is translated into a relational schema. In [11] we have shown that ER and EER schemas can be represented by relational schemas in Boyce-Codd Normal Form (BCNF), consisting of relation-schemes, key dependencies, referential integrity constraints, and null constraints. For example, following [11], the ER schema of figure $1(\mathrm{i})$ is represented by the $\mathrm{BCNF}$ schema $R S$ of figure 1(ii). Informally, object-sets are represented by relation-schemes, existence dependencies implied by object comections are represented by referential integrity constraints, and nullvalue restrictions on EER attributes are expressed by null (nulls-not-allowed) constraints. Regarding the goal of reducing the number of relations in a database, the question is whether a single relation-scheme can be used for representing multiple object-sets. 
Most ER and EER-oriented design methodologies recommend using a single relation-scheme for representing a binary many-to-one relationship-set and the entityset involved in that relationship-set with a many' cardinality. We have shown in [11] that methodologies such as that of [14] result in relational schemas that are inconsistent with the semantics of the corresponding ER or EER schema. Consider relational schema $R S^{\prime}$ of figure l(iii), which, following [14], represents the ER schema of figure 1(i). Then, contrary to the semantics of the ER structure of figure 1(i), $R S^{\prime}$ allows a wORKS relation to include tuples representing employees having a non-null assignment DATE, even if these employees are not working on any PROJECr (i.e. with a null NR value). Consequently, additional constraints need to be specified in order to ensure that the dacabase is consistent with the semantics of the corresponding ER schema. In relational schema $R S^{\prime}$, for example, relational attribute DATE should be con. strained to have a null value whenever attribute NR has a null value in a wORKS relation in order to represent accurately the association of ER attribute DATE with relationship-set wORKS; such restrictions can be expressed using null constraints.

In this paper we propose a relation merging technique for relational schernas consisting of relationschemes, key dependencies, referential integrity constraints, and nulls-not-allowed constraints. We do not consider alternative relational representations based on the Universal Relation assumptions [10], because their capability of expressing constraints is limited, and because their underlying assumptions cannot be maintained using commercial relational database management systems

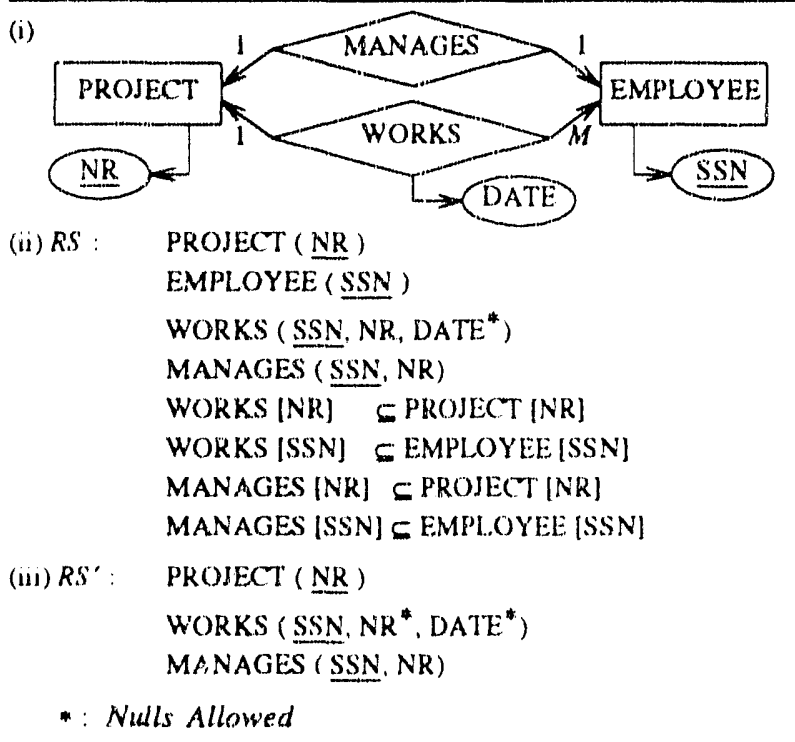

Fig. 1. Relational Schemas Representing an ER Schema.
(DBMS). We define a merging procedure that preserves the information-capacity and normal form of relational schemas. We show that in general merging requires the introduction of additional null construints for restricting the way in which null values anpear in merged relations. For example, relation-scheme wORKS of tigure l(iii) must be associated with null constraint DATE $\stackrel{\mathrm{E}_{\mathrm{X}}}{\rightarrow} \mathrm{NR}$ (read 'nonnull DATE requires non-null NR') in order to ensure that in a WORKS relation attribute DATE has a null value whenever attribute NR has a null value. It turns out that in certain cases only nulls-not-allowed constraints (i.e. constraints that restrict attributes to non-null values) are required. For example, relation-schemes EMPLOYEE and MANAGES of relational schema $R S$ of figure 1 (ii) can be merged into relation-scheme EMPLOYEE' (SSN, NR), where attribute SSN is not allowed to have null values, attribute NR is allowed to have null values, and no other null constraints need (o) be specified.

Employing effectively the merging procedure proposed in this paper depends on the capabilities of the underlying relational DBMS. An increasing number of commercial relational DBMSs support key dependencies, referential integrity constraints (which are key-based inclusion dependencies), and nulls-not-allowed constraints. For such systems we examine under what conditions the merging procedure does not require the introduction of additional constraints. In general, however, the merging technique presented in this paper may involve more complex null constraints and non key-based inclusion dependencies. Then the merging procedure can be: employed only if the underlying DBMS provides a mechanism for maintaining such constraints and dependencies, such as the triggers mechanism of SYBASE 4.0 [13] and the rules mechanism of INGRES 6.3 [6].

As mentioned above, relational schemas consisting of relation-schemes, key dependencies, referential integrity constraints, and null constraints may represent EER schemas [11]. Applying the merging procedure developed in this paper on relational schema trunslations of EER schemas shows that multiple object-sets are amenable for representation by a single relation-scheme not only for the standard binary many-10-one relationship-set case, but for more complex structures as well.

The paper is organized as follows. In section 2 we introduce the rebational concepts and notations used in this paper. The background for the merging technique is discussed in section 3 . The merging technique is developed in section 4. In section 5 we discuss several aspects of applying our merging technique to schemats of dalabases developed using commercial relational DBMSs, and to relational schema translations of EER schemas. We conclude the paper with a summary. 


\section{Preliminary Definitions}

We review briefly below the relational concepts used in this paper; details concerning these concepts can be found in textbooks such as [9].

A relation-scheme is a pair $R_{i}\left(X_{i}\right)$, where $R_{i}$ is a relation-scheme name and $X_{i}$ is a set of atuributes. Every attribute is associated with a domain, and every relationscbenc is cassociated with a relation consisting of tuples.

We denote by $t$ a tuple, and by $t[W]$ the subusple of $t$ corresponding to the attributes of $W$. A null value is denoted null, and a tuple consisting of $k$ null values is denoted null ${ }^{k}$. A tuple is said to be total iff it has only iron-null values. Two attributes are said to be compatible if they are associated with the same domain, and attribute sets $X$ and $Y$ are said to be compatible iff there exists a one-10-one correspondence of compatible attributes between $X$ and $Y$.

Let $R_{i}\left(X_{i}\right)$ be a relation-scheme associated with relation $r_{i}$, and let $W$ be a subset of $X_{i}$. The projection of $r_{i}$ on $W$ is denoted $\pi_{W}\left(r_{i}\right)$, and is equal to set of tuples $\left\{t\left[W_{i}\right] \mid t \in r_{i}\right\}$. The total projection of $r_{i}$ on $W$ is denoted $\pi \downarrow_{W}\left(r_{i}\right)$, and is equal to the subset of total tuples of $\pi_{W}\left(r_{i}\right)$. Renaming $W$ in $r_{i}$ to a sel of attributes $Y$ compatible with $W$ is denoted rename $\left(r_{i} ; W \leftarrow Y\right)$, and generates a relation associated with attribute set $\left(X_{i}-W\right) Y^{\prime}$, that is equal to set of tuples $\left\{t^{\prime} \mid t \in r_{i}\right.$, $t^{\prime}\left[X_{i}-W\right]=t\left[X_{i}-W\right]$, and $\left.t^{\prime}[Y]=t[W]\right\}$.

Let $R_{i}\left(X_{i}\right)$ and $R_{j}\left(X_{j}\right)$ be two relation-schemes associated with relations $r_{i}$ and $r_{j}$, respectively; let $Y$ and $Z$ be two compatible and disjoint subsets of $X_{i}$ and $X_{j}$, respectively; let $k_{i}$ and $k_{j}$ denote the number of attributes in $X_{i}$ and $X_{j}$, respectively. The equi-join of $r_{i}$ and $r_{j}$ on $(Y=Z)$ is denoted $r_{i} \underset{Y=Z}{\} r_{j}$, and is equal to set of tuples $\left\{t \mid t\left[X_{i}\right] \in r_{i}, t\left[X_{j}\right] \in r_{j}\right.$, and $\left.r[Y]=t[Z]\right\}$. The outer. equi-join of $r_{i}$ and $r_{j}$ on $(Y=Z)$ is denoted $r_{i} \underset{Y=Z}{\otimes}$, and is equal to the union of three relations, $r_{1}, r_{2}$, and $r_{3}$, where: $r_{1}=r_{i} \bigotimes_{Y=Z} r_{j}, r_{2}=\left\{l \mid c\left[X_{i}\right]=n u \|^{k_{i}}, \quad t\left[X_{j}\right] \in r_{j}\right.$, and $Z t^{\prime} \in r_{j}$ s.t. $\left.t^{\prime}[Y]=t[Z]\right\}$, and $r_{3}=\left\{t \mid t\left[X_{i}\right] \in r_{i}\right.$, $t\left[X_{j}\right]=n u l l^{k_{j}}$, and $Z t^{\prime \prime} \in r_{j}$ s.t. $\left.:[Y]=t^{\prime \prime}[Z]\right\}$.

Let $R_{i}\left(X_{i}\right)$ be a relation-scheme associared with relation $r_{i}$. A functional deperdency over $R_{i}$ is a statement of the form $R_{i}: Y \rightarrow Z$, where $Y$ and $Z$ are subsets of $X_{i}: R_{i}: Y \rightarrow Z$ is satisfied by $r_{i}$ iff for every two tuples of $r_{i}, t$ and $t^{\prime}, t[Y]=t^{\prime}[Y]$ implies $t\left[Z j=t^{\prime}[Z]\right.$. A kiey associated with $R_{i}$ is a subset of $X_{i}, K_{i}$, such that $R_{i}: K_{i} \rightarrow X_{i}$ is satisfied by every $r_{i}$ associated with $R_{i}$ and there does not exist any proper subset of $K_{i}$ having this property. A relation-scheme can be associated with several candidate keys from which one primary key is chosen. If all functional dependencies associated with $R_{i}$ involve in their left-hand sides supersets of keys, then $R_{i}$ is said to bx in Boyce-Codd Normal Form (BCNF).

Let $R_{i}\left(X_{i}\right)$ and $R_{j}\left(X_{j}\right)$ be two relation-schernes associated with relations $r_{i}$ and $r_{j}$, respectively. An inclusion dependency is a statement of the form $R_{i}[Y] \subseteq R_{j}[Z]$, where $Y$ and $Z$ are compatible subsets of $X_{i}$ and $X_{j}$, respectively; $R_{i}[Y] \subseteq R_{j}[Z]$ is satisfied by $r_{i}$ and $r_{j}$ iff $\pi \downarrow_{Y}\left(r_{i}\right) \subseteq \pi \downarrow_{Z}\left(r_{j}\right)$. If $Z$ is the primary key of $R_{j}$ then $R_{i}[Y] \subseteq R_{j}[Z]$ is said to be key-based, and $Y$ is called a foreign key in $R_{i}$. Key ubsed inclusion dependencies are usually called referential integrity constraints [4].

A relational schema $R S$ is a pair $(R, \Delta)$, where $R$ is a set of relation-schemes and $\Delta$ is a set of dependencies and constraints over $R$. A database state $r$ of (associated with) $R S$ consists of the relations associated with the relation-schemes of $R$; state $r$ is said to be consistent iff it satisfies the dependencies and constraints of $\Delta$.

It is well known in database design that the same data can be structured in different ways, that is, represented by different schemas, proviled that these schemas have equivalent information-capacities [8]. We are interested only in relational schemas that preserve the attribute values. This requirement is captured by the information-capacity equivalence defired below, which follows the definition of generic equivalence of [8].

Definition 2.1. 'Two relational schemas, $R S$ and $R S^{\prime}$, are said to have equivalent information-capacity iff there exist total functions $\phi$ and $\phi^{\prime}$ such that:

1. $\phi$ maps consisten database states of $R S$ into consistent database states of $R S^{\prime}$;

2. $\phi^{\prime}$ maps consistent database states of $R S^{\prime}$ into consistent database states of $R S$;

3. the composition of $\phi$ followed by $\phi^{\prime}$ is the identity on the set of all consistent database states of $R S$; the composition of $\phi^{\prime}$ followed by $\phi$ is the identity on the set of all consistent database states of $R S$;

4. For any database state $r$ of $R S, \phi$ preserves the data values of $r^{\dagger}$; similarly, for any chatabase state $r^{\prime}$ of $R S^{\prime}, \phi^{\prime}$ preserves the data values of $r^{\prime}$.

Informally, a schema $R S^{\prime}$ has equivalent information-capacity with a schema $R S$, if $R S^{\prime}$ can bx associated with the same number of database states as $R S$; that is, not only every legal database state associated with $R S$ must be exactly reconstructed from its mapping into a database state of $R S^{\prime}$, but every database state associated with $R S^{\prime}$ must be mappable into a database state of $R S$.

\footnotetext{
${ }^{\dagger}$ A dalabase state mapping $\phi$ is sad to preserve the data values of a database state $r$ iff the values of $\phi(r)$ are included in $r$.
} 


\section{Background for Merging Relations}

In this section we examine the main aspects of the merging technique developed in this paper, and introduce the null constraints involved in this technique.

In a relational database, real-world objects are represented by tuples and are identified by primary-key values. We assume that every relation in a database represents a homogeneous set of objects, and that relations associated with compatible primary-keys represent semantically compatibie sets of real-world objects. Accordingly, in order to avoid creating relations that may represent heterogeneous sets of semantically incompatible objects, we consider for merging only relation-schemes that are associated with pairwise compatible primary-keys.

Let $\bar{R}$ denote a set of relation-schemes largeted for merging, and let $\bar{r}$ denote the set of relations associated with the relation-schemes of $\bar{R}$. Merging must preserve the tuples contained in the relations of $\bar{r}$, and therefore if involves outer-equi-joining on (compatible) prixnary-keys the relations of $\vec{r}$. However, instead of being joined directly, the relations of $\bar{r}$ are outer-equi-joined with a key--relation that contains all the primary-key values appearing in the relations of $\bar{r}$. The result of an outerequi-join may contain redundant attribute values that must be subsequently removed. Consequenty, the merging technique developed in the next section involves:

1. outer-equi-joining a key-relation with the relations involved in merging; and

2. projecting out the redundant attribute values from the result of the outer-equi-join above.

The key-relation is defined below.

Definition 3.i. Let $R S=(R, \Delta)$ be a relational schema, and let $\bar{R}$ be a subsei of $R$ consisting of relation-schemes that have pairwise compatible primary-keys. A relationscheme $R_{k}\left(X_{k}\right)$ is said is be a key-relation of $\bar{R}$ iff (i) $R_{k}$ has it primary-key, $K_{k}$, that is pairwise compatible with eact. of the primary-keys of the relation-schemes in $\bar{R}$, and (ii) for every database state assiciated with $R S$, the relation associated with $R_{k}, r_{k}$, satisfies the following condition: $\pi_{K_{t}}\left(r_{k}\right)=\bigcup_{R_{i} \in \bar{R}}\left(\right.$ rename $\left.\left(\pi_{K_{i}}\left(r_{i}\right), K_{i} \leftarrow K_{k}\right)\right)$.

We consider in this paper relational schemas consisting of relation-schemes, key dependencies, key-based inclusion dependencies, and null constraints. We show below that for such schemas the key-relation of $\bar{R}$ can be onc of the relation-scithemes of $\bar{R}$.

Proposition 3.1. Let $R S=(R, F \cup I \cup N)$ be a relational schema, where $R, F, I$, and $N$ denote sets of relationschemes, key dependencies, key-based inciusion dependencies, and null constraints, respectively. Let $\bar{R}$ be a subset of $R$ consisting of relation-schemes that have pairwise compatible primary-keys. Let $R_{0}$ belong to $\bar{R}$, and let sets Refkey $\left(R_{o}, \bar{R}\right)$ and Refkey ${ }^{\circ}\left(R_{o}, \bar{R}\right)$ be defined recursively as follows:

- Refkey $\left(R_{o}, \bar{R}\right)=\left\{R_{i} \mid R_{i} \in \bar{R}, R_{i}\left[K_{i}\right] \subseteq R_{o}\left[K_{o}\right] \in I\right\}$;

- Refkey ${ }^{*}\left(R_{o}, \bar{R}\right)=\operatorname{Refkey}\left(R_{o}, \bar{R}\right)$

$$
\cup_{R_{i} \in \operatorname{Refkey}\left(R_{m}, \ddot{R}\right)} \operatorname{RefRey^{*}}\left(R_{i}, \bar{R}\right)
$$

Then $\pi_{K_{0}}\left(r_{o}\right)=\cup_{R_{i} \in \bar{R}}\left(\right.$ rename $\left.\left(\pi_{K^{\prime}}\left(r_{i}\right), K_{i} \leftarrow K_{o}\right)\right)$ for every database state associated with $R S$,

iff $\bar{R}=\left\{R_{o}\right\} \cup$ Refkey $^{*}\left(R_{o}, \bar{R}\right)$.

Proof Sketch. Straightforward, following the definition of inclusion dependencies.

Note that if a set of relation-schemes $\bar{R}$ defined as above does not contain a key-relation, then a new relation-scheme, $R_{k}\left(K_{k}\right)$, can be specified as a key-relation of $\bar{R}$, that is, so that $K_{k}$ is pairwise compatible with each of the primary-keys of the relation-schemes of $\bar{R}$, and so that for every database state associated with relational schema $R S, R_{k}$ is associated with relation relation $r_{k}$, where $r_{k}:=\cup_{R_{i} \in \bar{R}}\left(\right.$ rename $\left.\left(\pi_{K_{i}}\left(r_{i}\right), K_{i} \leftarrow K_{k}^{\prime}\right)\right)$.

For example, let $\vec{R}$ consist of relation-schemes OFFER and TEACH of figure 2, and let $r_{O}$ and $r_{T}$ be relations associated with OFFER and TEACH, respectively. A relation-scheme $R_{C}$ can be a key-relation of $\bar{R}$ if the primary-key of $R_{C}$, say $\mathrm{CN}$, is pairwise compatible with O.CN and T.CN, and if the relation associated with $R_{C}, r_{C}$, satisfies the following condition: $\pi_{\mathrm{CN}}\left(r_{C}\right)=$ rename $\left(\pi_{\mathrm{OCN}}\left(r_{O}\right), 0 . \mathrm{CN} \leftarrow \mathrm{CN}\right) \cup$ rename $\left(\pi_{\mathrm{T} . \mathrm{N}}\left(r_{O}\right), \mathrm{T} . \mathrm{CN} \leftarrow \mathrm{CN}\right)$. Then merging relation-schemes OFFER and TEACH into a new relation-scheme ASSIGN (see figure 2 ) involves a state mapping defining the relation associated with Assicin: $r_{\mathrm{A}}:=r_{C_{\mathrm{CN}}=\mathrm{OCN}} r_{O} \underset{\mathrm{CN}=\mathrm{TCN}}{\otimes} r_{T}$. Note that if relationschemes OFFER and TEACH are not involved in any inclusion dependency, then attributes $O . C N$ and T.CN are redundant in ASSIGN; however, if OFFER and TEACH are involved in the right-hand sides of two distinct inclusion dependencies, then these attributes are not redundamt in ASSIGN. If relation-schemes OFFER and TEACH are involved in inclusion dependency TEACH[T.CN] $\subseteq$ JFFE $[O . C N]$ then, following proposition 3.1, OFFER is a key-relation of $\bar{R}$, and merging OFFER with TEACH involves outer-equi-joining relations $r_{0}$ and $r_{T}$.

$$
\begin{aligned}
& \bar{R}=\{\text { OFFER }(\text { O.CN, ODN }), \text { TEACH }(\text { T.CN }, T . F N)\} \\
& \text { - Merge } \rightarrow \text { ASSIGN (CN O.CN, ODN, T.CN, THN) }
\end{aligned}
$$

Fig. 2. Relation-Scheme Examples (keys are :nderlined). 
The result of an outer-equi-join is a relation, $r_{m}$, that usually contains null values; these null values must be restricted in order to ensure that the joined relations can be reconstructed from $r_{m}$ without losing or adding information. Such restrictions are called null constraints and are defined below. A null constraint is a single-tuple restriction on where and how nulls should appear in a relation [9]. In the following definitions $R_{i}\left(X_{i}\right)$ denotes a relationscheme, and $r_{i}$ denotes a relation associated with $R_{i}\left(X_{i}\right)$.

A null-existence constraint is a statement of the form $R_{i}: Y \stackrel{E_{X}}{\rightarrow} Z$, where $Y$ and $Z$ are subsets of $X_{i}$; $R_{i}: Y \stackrel{\mathbf{E}_{\mathrm{X}}}{\rightarrow} Z$ is satisfied by $r_{i}$ iff for every tuple $t$ of $r_{i}$, $t[Y]$ is total only if $t[Z]$ is total. A nulls-not-allowed constraint is a null-existence constraint of the form $R_{i}: \varnothing \stackrel{\mathbb{x}_{\mathbf{x}}}{\rightarrow} Z$, that is satisfied iff every subtuple $t[Z]$ of $r_{i}$ is total. In relations associated with relation-scheme ASSIGN of figure 2, for example, AsSIGN: T.CN $\stackrel{\mathrm{Ex}^{\circ}}{\rightarrow}$ O.CN does not allow tuples that contain null $O . C N$ values together with non-null T.CN values, while ASSIGN: $\stackrel{\mathrm{Ex}^{\mathrm{X}}}{\rightarrow}$ O.CN does not allow tuples containing null $\mathrm{O} . \mathrm{CN}$ values.

A null-synchronization set is a set of null-existence constraints, of the form $\left\{R_{i}: A_{j} \stackrel{\mathrm{Ex}_{x}}{\rightarrow} Y \mid A_{j} \in Y\right\}$, denoted $R_{i}: N S(Y) ; R_{i}: N S(Y)$ is satisfied by $r_{i}$ iff for every tuple $t$ of $r_{i}, t[Y]$ is either total or consists entirely of null values (i.e. cannot be partly null). Consider relationscheme ASSIGN of figure 2; the two null-existence constraints of ASSIGN:NS (T.CN, T.FN) do not allow in relations associated with ASSIGN tuples $t$ containing partly null I [T.CN, T.FN] subtuples.

A part-null constraint is a statement of the form $R_{i}: P N\left(Y_{1}, \ldots, Y_{m}\right)$, where $Y_{j}, 1 \leq j \leq m$, is a subset of $X_{i}$; $R_{i}: P N\left(Y_{1}, \ldots, Y_{m}\right)$ is satisfied by $r_{i}$ iff for every tuple $t$ of $r_{i}$, at least one subtuple $t\left[Y_{j}\right], 1 \leq j \leq m$, is total. In relations associated with relation-scheme ASSIGN of figure 2 , for example, ASSIGN: PN ([O.CN, O.FN), (T.CN. T.FN)) ensures that in every tuple $t$ of such relations either subtuple $l$ [T.CN, T.FN], or subtuple $t[\mathrm{O} . \mathrm{CN}, \mathrm{O.FN}]$, or both are total.

Finally, a total-equality constraint is a statement of the form $R_{i}: Y=\downarrow Z$, where $Y$ and $Z$ are compatible subsets of $X_{i} ; R_{i}: Y=\downarrow Z$ is satisfied by $r_{i}$ iff for every luple $t$ of $r_{i}, t[Y]=t[Z]$ whenever both $t[Y]$ and $t[Z]$ are total. Consider again relation-scheme ASSIGN of figure 2; total-equality constraint ASSIGN : T.CN $=\downarrow$ O.CN ensures that in every tuple of relations associated with ASSIGN, non-null values for attributes T.CN and $O . C N$ are equal.

Inference axioms for null-existence constraints have the form of the inference axiorns for functional dependencies (see [9]). Inference axioms for total-equality constraints are analogous to the inference axioms for the equality constraints of [7]. Null-existence, total-equality, and part-null constraints do not interact with each other.

\section{A Relation Merging Technique}

In this section we develop a relation merging technique that preserves the information-capacity and normal form of the relational schemas on which it is applied.

\subsection{Merging Relation-Schemes}

We define below a procedure for merging relationschemes in relational schemas of the form $(R, F \cup I \cup N)$, where $R, F, I$, and $N$ denote sets of relation-schemes, key dependencies, key-based inclusion dependencies, and null constraints, respectively; such a schema is shown in figure 3 . We assume that the attributes are assigned globally unique names in the schema. Let $\vec{R}$ be a subset of $R$ consisting of relation-schemes associated with pairwise compatible primary-keys; merging the relation-schemes of $\bar{R}$ implies mapping $(R, F \cup l \cup N)$ into a new schema, $\left(R^{\prime}, F^{\prime} \cup I^{\prime} \cup N^{\prime}\right)$, where $R^{\prime}$ results by replacing the relation-schernes of $\bar{R}$ with a new relation-scheme, $R_{m}$, and $F^{\prime}, I^{\prime}$, and $N^{\prime}$ consist of adjusted key dependencies, inclusion dependencies, and null constraints, respectively. For the sake of simplicity, we assume that initially the attributes associated with relation-schemes of $\vec{R}$ are not allowed to have null values.

Definition 4.1. Let $R S=(R, F \cup I \cup N)$ be a relational schema, where $R, F, l$, and $N$ denote sets of relationschemes, key dependencies, key-based inclusion dependencies, and null constraints, respectively. Let $\bar{R}$ be a subset of $R$ consisting of relation-schemes associated with pairwise compatible primary-keys, so that every relationscheme $R_{i}\left(X_{i}\right)$ of $\vec{R}$ is associated with nulls-not-allowed constraint $R_{i}: \varnothing \stackrel{\mathbb{E}_{M}}{\rightarrow} X_{i}$. Let $R_{k}\left(X_{k}\right)$ be a relation-scheme

\section{Relation-Schemes (underlined keys)}
(1) PERSON (P.SSN)
(5) DEPARTMENT (D.NAME)
(2) FACULTY (F.SSN)
(6) OFFER (O.C.NR, O.D.NAME)
(3) STUDENT (S.SSN)
(7) TEACH (T.C.NR, T.F.SSN)
(4) COLRSE (C.NR)
(8) A.SSIST (A.CNR, A.S.SSN)

Inclusion Dependencies

\begin{tabular}{ll} 
(1) FACULTY [F.SSN] & \multicolumn{1}{c}{ PERSON [P.SSN] } \\
(2) STUDENT [S.SSN] & ᄃ PERSON [P.SSN] \\
(3) OFFER [O.C.NR] & ᄃ COURSE [C.NR] \\
(4) OFFER [O.D.NAME] & 5 DEPARTMENT [D.NAME] \\
(5) TEACH [T.C.NR] & 5 OFFER [O.C.NR] \\
(6) TEACH [T.F.SSN] & E FACULTY [T.SSN] \\
(7) ASSIST [A.C.NR] & 5 OFFER [O.C.NR] \\
(8) ASSIST [A.S.SSN] & 5 STUDENT [S.SSN]
\end{tabular}

Null ( mulls-not-allowed) Constraints

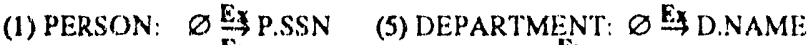

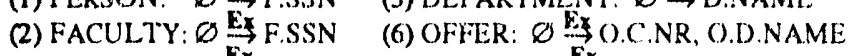

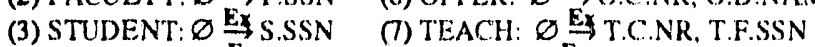

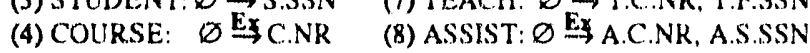

Abbreviations: $\quad A=A S S I S T, C=C O U R S E, D=D E P A K T M E N T$, $\mathrm{F}=$ FACULTY, $($ )=OFFER, S=STUDENT, T $=$ TEACH

Fig. 3. A Relational Schema. 
defined as follows: if $\bar{R}$ contains a key-relation, $R_{o}$, then $R_{k}:=R_{o}, X_{k}:=X_{o}$, and $K_{k}:=K_{o}$; otherwise $X_{k}=K_{k}$, where $K_{k}$ is disjoint with the attribute sets associated with the relation-schemes of $\vec{R}$, and for every database state associated with $R S, R_{k}$ is associated with relation $r_{k}$, where $r_{k}:=\cup_{R_{l} \in \bar{R}}\left(\right.$ rename $\left.\left(\pi_{K_{i}}\left(r_{i}\right), K_{i} \leftarrow K_{k}\right)\right)$.

Merge $(\bar{R})$ applied on $R S$ generates relational schema $R S^{\prime}=\left(R^{\prime}, F^{\prime} \cup I^{\prime} \cup N^{\prime}\right)$ as follows:

1. $R^{\prime}$ results by replacing in $R$ the relation-schemes of $\bar{R}$ with a new relation-scheme, $R_{m}\left(X_{m}\right)$, such that $K_{m}:=K_{k}$ and $X_{m}:=X_{k} \cup_{R_{1}\left(X_{i}\right) \in \bar{R}} X_{i}$;

2. $F^{\prime}$ results by replacing the key dependencies involving primary-keys associated with the relation-schemes of $\bar{R}$ with key dependency $R_{m}: K_{m} \rightarrow X_{m}$;

3. $N^{\prime}$ is generated as follows:

a. the nulls-not-allowed constraints associated with the relation-schemes of $\bar{R}$ are replaced with nulls-notallowed constraint $R_{m}: \varnothing^{\mathrm{Ex}} \rightarrow X_{k}$;

b. for every relation-scheme $R_{i}\left(X_{i}\right)$ of $\bar{R}$, if $K_{i} \neq K_{m}$, where $K_{i}$ is the primary-key of $R_{i}$, then totalequality constraint $R_{m}: K_{m}=\downarrow K_{i}$ is added to $N^{\prime}$;

c. for every relation-scheme $R_{i}\left(X_{i}\right)$ of $\left(\bar{R}-\left\{R_{k}\right\}\right)$, if $X_{i}$ consists of more than one attribute, then the null-existence constraints of null-synchronization set $R_{m}: N S\left(X_{i}\right)$ are added to $N^{\prime}$;

d. if $R_{k}$ does not belong to $\bar{R}$, then part-null constraint $R_{m}: P N\left(X_{1}, \ldots, X_{n}\right)$ involving the attribute sets associated with relation-schemes $R_{i}\left(X_{i}\right)$ of $\bar{R}$, $1 \leq i \leq n$, is added to $N^{\prime}$;

e. for every inclusion dependency of $I$ of the form $R_{j}[Z] \subseteq R_{i}\left[K_{i}\right]$, where $R_{i}$ and $R_{j}$ belong to $\bar{R}$, if $K_{i} \neq K_{m}^{\prime}$ then null-existence constraint $R_{m}: X_{j} \stackrel{\mathbb{E x}_{x}}{\rightarrow} X_{i}$ is added to $N^{\prime}$;

4. $I^{\prime}$ results by (a) replacing $R_{i}$ with $R_{m}$ in every inclusion dependency of $I$ involving a relation-scheme $R_{i}$ of $\bar{R}$; (b) replacing $K_{i}$ with $K_{m}$ in every inclusion dependency of $l^{\prime}$, of the form $R_{m}[Z] \subseteq R_{m}\left[K_{i}\right]$; and (c) removing from $I^{\prime}$ inclusion dependencies of the form $R_{m}\left[K_{i}\right] \subseteq R_{m}\left[K_{m}\right]$, where $K_{i}$ is the primarykey of a relation-scheme of $\vec{R}$.

Merge $(\bar{R})$ is associated with two state mappings, $\eta$ and $\eta^{\prime}$, where $\eta$ maps a database state $r$ of $R S$ into a database state $r^{\prime}$ of $R S^{\prime}$, and $\eta^{\prime}$ mans a chatabase state $r^{\prime}$ of $R S^{\prime}$ into a database state $r$ of $R S$, as follows:

$\eta$ is identity for relations of $r$ associated with relationschemes of $(R-\bar{R})$; and maps set of relations $\bar{r}=$ $\left\{r_{1} \mid r_{i} \in r, r_{i}\right.$ is associated with $R_{i}$ of $\left.\bar{R}\right\}$ into $r_{m}$ as follows: (i) $r_{m}:=r_{k}$; (ii) for each $R_{i}$ of $\left(\bar{R}-\left\{R_{k}\right\}\right)$

$$
\text { do } r_{m}:=r_{m} \underset{K_{m}=K_{i}}{\stackrel{O}{R_{i}}} r_{i}
$$

$\eta^{\prime}$ is identin for relations of $\left(r^{\prime}-r_{m}\right)$; and maps relation $r_{m}$ into relations $\tilde{r_{i}}:=\pi \downarrow_{X_{i}}\left(r_{m}\right)$, where $X_{i}$ is the attribute set of a relation-scheme $R_{i}\left(X_{i}\right)$ of $\bar{R}$.

Merging the relation-schemes of $\bar{R}$ into a new relation-scheme, $R_{m}$, involves defining the relation associated with $R_{m}, r_{m}$, as the outer-equi-join of a key-relation with the relations of $\bar{r}$, where $\bar{r}$ consists of relations associated with relation-schemes of $\bar{R}$. The set of dependencies and constraints generated by merging ensure that the relations of $\bar{r}$ can always be reconstructed (by total projection) from $r_{m}$. Thus, the total-equality constraints require the tuples of $r_{m}$ to satisfy the join conditions involved in the definition of $r_{m}$; attributes that are not associated with the key-relation are allowed to have null values in $r_{m}$; the null-existence constraints of a given null-syichronization set ensure that in every tuple of $r_{m}$, a subtuple (of the form $\left.t\left[X_{i}\right]\right)$ corresponding to a tuple of a relation of $\bar{r}$ cannot have both null and non-null values; the part-null constraint ensures that in every tuple of $r_{m}$ there is at least one total subtuple corresponding to a tuple of a relation of $\bar{r}$; the (inner-relational) null-existence constraints generated in step 3(e) express the inter-relational existence constraints implied by the inclusion dependencies involving pairs of relation-schemes of $\bar{R}$. Finally, the key dependencies removed in step 2 and the inclusion dependencies removed in step 4(c), are implied by the new key dependency and total-equality constraints, respectively by the total-equality and null-existence constraints (generated in step 3(e)), and therefore are redundant.

Two examples of applying Merge on the relational schema of tigure 3 are shown in figures 4 and 5 ; in both examples the key-relation is relation-scheme COURSE. While in the example shown in figure 4 merging involves only relation-schemes COURSE, OFFER, and TEACH, in the example shown in figure 5 merging involves an additional relation-scheme, ASsIST. The following proposition shows that Merge preserves the information-capacity (in the

Relation-Schemes 4,6 , and 7 are replaced by COURSE' (C.NR, O.C.NR, O.D.NAME, T.C.NR, T.F.SSN)

Inclusion Dependencies 3 to 7 are replaced by (9) : COURSE' [O.D.NAME] \& DEPARTMENT [D.NAME]

(10): COURSE' [T.F.SSN] GFACULTY [F.SSN]

(11): ASSIST [A.C.NR] GCOURSE' [O.C.NR]

Null Constraints 4,6 , and 7 are replaced by the

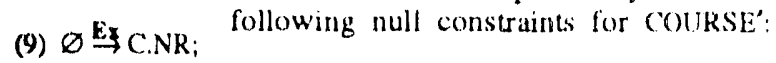

(10) NS (O.C.NR, O.D.NAME): (11) NS (T.C.NR, T.F.SSN);

(12) T.C.NR,T.F.SSN $\stackrel{\text { E}}{\rightarrow}$ O.C.NR,O.D.NAME;

(13) C.NR $=\downarrow$ O.C.NR; (14) C.NR $=\downarrow$ T.C.NR

Fig. 4. Applying on Relational Schema of Fig. 3 Merge (COURSE, OFFER, TEACH). 
sense of definition 2.1) and the normal form of the schemas on which it is applied.

Proposition 4.1. Let $R S, R S^{\prime}, \bar{K}$, and Merge be defined as in definition 4.1, so that $R S^{\prime}=\left(R^{\prime}, F^{\prime} \cup l^{\prime} \cup N^{\prime}\right)$ is the result of applying Merge $(\bar{R})$ on $R S=(R, F \cup I \cup N)$. Then (i) $R S$ and $R S^{\prime}$ have equivalent informationcapacities; and (ii) the relation-schemes of $R^{\prime}$ are in BCNF. Proof Sketch. (i) The proof refers to the conditions of definition 2.1, and regards only the relations affected by merging; for the first two conditions, the proof follows the definition of $\eta$ and $\eta^{\prime}$; for the third condition, the proof follows from the fact that Merge preserves the primarykeys associated with the relation-schemes of $\bar{R}$, and that the outer-equi-joins involved in $\eta$ are on primary keys; the last condition is obviously satisfied. (ii) The proof that all functional dependencies implied by $\left(F^{\prime} \cup I^{\prime} \cup N^{\prime}\right)$ are key dependencies is based on the fact that the closure of $F$ can be computed independently of $I$ (see [3]), and on the inference axioms for total-equality constraints and functional dependencies (see [7]).

The attributes involved in the total-equality constraints generated by Merge seem to be removable without any effect on the information-capacity cf the relational schema. Since Merge preserves the normal form (BCNF) of relational schemas, these potentially redundant attributes are not a source of update anomalies [9]. Removing redundant altributes, however, simplifies the set of null constraints associated with merged relationschemes, as well as reduces the size of the relations associated with merged relation-schemes. A procedure for removing redundant attributes is specified below.

\subsection{Removing Redundant Attributes}

We define below the conditions characterizing redundant attributes in relation-schernes generated by Merge, and then specify a procedure for removing such attributes.

Relation-Schemes $4,6,7$, and 8 are replaced by :

COURSE:" C.NR, O.C.NR, O.D.NAME, T.C.NR, T.F.SSN, A.C.NR, A.S.SSN)

Inclusion Dependencles 3 to 8 are replaced by:

(9) : COURSE" [O.D.NAME] CDEPARTMENT [D.NAME]

(10): COURSE" [T.F.SSN] E FACULTY [F.SSN]

(11): COURSE" [A.S.SSN] ESTUDENT [S.SSN]

Null Constraints $4,6, \%$, and 8 are replaced by the

(9) $\varnothing \stackrel{\text { EX }}{\rightarrow}$ C.NR; following null constraints for COURSE":

(11) NS (T.C.NR, TF.SSN); (10) NS (O.C.NR, O.D.NAME),

(13) T.C.NR, J.F.SSN $\stackrel{\text { Eq}}{\rightarrow}$ O.C.NR,O.D.NAME;

(14) A.C.NR,A.S.SSN 焉O.C.NR,O.D.NAME;

(15) C.NR $=\downarrow$ O.C.NR; (16) C.NR $=\downarrow$ T.C.NR; (17) C.NR $=\downarrow$ A.C.NR

Fig. 5. Applying on Relational Schema of Fig. 3 Merge (COURSE, OFFER, TEACH, ASSIST).
Definition 4.2. Let $R S, R S^{\prime}, \bar{R}$, and Merge be defined as in definition 4.1, so that $R S^{\prime}=\left(R^{\prime}, F^{\prime} \cup I^{\prime} \cup N^{\prime}\right)$ is the result of applying Merge $(\bar{R})$ on $R S$, and $R_{m}\left(X_{m}\right)$ is the result of merging the relation-schemes of $\bar{R}$. Let $X_{i}$ be a subset of $X_{m}$, such that $X_{i}$ is associated with relationscheme $R_{i}$ of $\bar{R}$, and let $X_{i}$ be a subset of $X_{i}$ involved in a total-equality constraint associated with $R_{m}$, such that $Y_{i} \neq K_{m}$. Then $Y_{i}$ is said to be removable in $R_{m}$ if the following conditions are satisfied:

(1) $\left|X_{i}-Y_{i}\right| \geq 1$.

(2) $Y_{i}$ is not involved in the right-hand side of $I^{\prime}$ inclusion dependencies of the form $R_{j}[Z] \subseteq R_{m}\left[Y_{i}\right], R_{j} \neq R_{m}$.

(3) If $Y_{i}$ is involved in the left-hand side of an $I^{\prime}$ inclusion dependency of the form $R_{m}\left[Y_{i}\right] \subseteq R_{j}\left[K_{j}\right], R_{j} \neq R_{m}$, then for every subset of $X_{m}, W$, involved in a totalequality constraint associated with $R_{m}, J^{\prime}$ includes an inclusion dependency of the form $R_{m}[W] \subseteq R_{j}\left[K_{j}\right]$.

(4) $Y_{i}$ does not overlap with other foreign-keys of $R_{m}$, that is, if attributes of $Y_{i}$ are involved in the left-hand side of an $I^{\prime}$ inclusion dependency of the form $R_{m}[W] \subseteq R_{j}\left[K_{j}\right], r_{j} \neq R_{m}$, then $W \equiv Y_{i}$.

For example, O.C.NR, T.C.NR, and A.C.NR are removable attributes in relation-scheme COURSE" of figure 5.

Note that an attribute that is removable in a merged relation-scheme associated with aturibute set $X_{m}$, is not necessarily removable in a merged relation-scheme associated with a proper subset of $X_{m}$. Let $R_{m}\left(X_{m}\right)$ and $R_{m}^{\prime}\left(X_{m}^{\prime}\right)$ result by applying $\operatorname{Merge}(\bar{R})$ and $\operatorname{Merge}\left(\bar{R}^{\prime}\right)$, respectively, on a relational schema $R S$, where $R^{\prime}$ is a subset of $R$. Then $X^{\prime}{ }_{m}$ is a subset of $X_{m}$, but because of condition (2) of definition 4.2, a cornmon subset of $X_{m}$ and $X_{m}^{\prime}$ can be removable in $R_{m}$, but not in $R_{m}^{\prime}$. For exam. ple, while attribute O.C.NR is removable in relation-scheme COURSE" of figure 5, O.C.NR is not removable in relationscheme COURSE' of figure 4.

Definition 4.3. Let $R S, R S^{\prime}, \bar{R}$, and Merge be defined as in definition 4.1, so that $R S^{\prime}=\left(R^{\prime}, F^{\prime} \cup I^{\prime} \cup N^{\prime}\right)$ is the result of applying Merge $(\bar{R})$ on $R S$, and $R_{m}\left(X_{m}\right)$ is the result of merging the relation-schemes of $\bar{R}$. Let $Y_{i}$ be a subset of $X_{m}$, removable in $R_{m}$. Remove $\left(Y_{i}\right)$ applied on $R S^{\prime}$ generates $R S^{\prime \prime}=\left(R^{\prime \prime}, F^{\prime \prime} \cup I^{\prime \prime} \cup N^{\prime \prime}\right)$ as follows:

1. $R^{\prime \prime}$ results by removing the attributes of $Y_{i}$ from attribute set $X_{m}$ associated with $R_{m}$;

2. $F^{\prime \prime}$ results by replacing in key dependencies of $F^{\prime}$ every attribute $A$ of $Y_{i}$ with an attribute of $K_{m}$ that corresponds to $A$ in a total-equality constraint of $N^{\prime}$;

3. $I^{\prime \prime}$ results by replacing $Y_{i}$ with $K_{m}$ in inclusion dependencies of $I^{\prime}$ of the form $R_{m}\left[Y_{i}\right] \subseteq R_{j}\left[K_{j}\right]$;

4. $N^{\prime \prime}$ results by removing (a) the attributes of $Y_{i}$ from the part-null and null-existence constraints of $N^{\prime}$, and 
(b) total-equality constraint $R_{m}: K_{m}=\downarrow Y_{i}$ from $N^{\prime}$.

Remove $\left(Y_{i}\right)$ is associated with two state mappings, $\mu$ and $\mu^{\prime}$, where $\mu$ maps a database state $r^{\prime}$ of $R S^{\prime}$ into a database state $r^{\prime \prime}$ of $R S^{\prime \prime}$, and $\mu^{\prime}$ maps a database state $r^{\prime \prime}$ of $R S^{\prime \prime}$ into a database state $r^{\prime}$ of $R S^{\prime}$, as follows:

$\mu$ is identity for relations of $r^{\prime}$ associated with relation-schemes of $\left(R^{\prime}-\left\{R_{m}\right\}\right)$; and maps relation $r_{m}^{\prime}$ associated with $R_{m}$ into $r_{m}^{\prime \prime}:=\pi_{\left(X_{m}-Y_{i}\right)}\left(r_{m}^{\prime}\right)$;

$\mu^{\prime}$ is identity for relations of $r^{\prime \prime}$ associated with relation-schemes of $\left(R^{\prime \prime}-\left\{R_{m}\right\}\right)$; and maps relation $r_{m}^{\prime \prime}$ associated with $R_{m}$, into $r_{m}^{\prime}:=r_{m}^{\prime \prime} K_{m}=Y_{l}$ ( rename $\left(\pi_{K_{m}}\left(\pi \downarrow_{K_{m}\left(X_{i}-Y_{i}\right)}\left(r^{\prime \prime}{ }_{m}\right)\right), K_{m} \leftarrow Y_{i}\right)$ ).

An example of applying Remove is shown in figure 6, where attributes O.C.NR, T.C.NR, and A.C.NR are successively removed from relation-scheme cOURSE" of figure 5. The following pi sposition shows that Remove preserves the information-capacity (in the sense of definition 2.1) of the relational schemas on which it is applied.

Proposition 4.2. Let $R S, R S^{\prime}, \bar{R}$, and Merge be defined as in definition 4.1, so that $R_{m}\left(X_{m}\right)$ in $R S^{\prime}$ is the result of merging the relation-schemes of $\bar{R}$. Let $R S^{\prime \prime}, Y_{i}$, and Remove be defined as in definition 4.3, so that $Y_{i}$ is a removable subset of $X_{m}$, and $R S^{\prime \prime}$ is the result of applying Remove $\left(Y_{i}\right)$ on $R S^{\prime}$. Then $R S^{\prime}$ and $R S^{\prime \prime}$ have equivalent information-capacities.

Proof Sketch. The proof regards only the relations (associated with $R_{m}$ ) affected by removal, and refers to the conditions of definition 2.1. The last condition is obviously satisfied. For the first two conditions, the proof follows the definition of the state mappings; note that replacing inclusion dependencies of the form $R_{m}\left[Y_{i}\right] \subseteq R_{j}\left[K_{j}\right]$ with $R_{m}\left[K_{m}\right] \subseteq R_{j}\left[K_{j}\right]$ can be accomplished because of conditions (3) and (4) of definition 4.2. For the third condition, the proof follows from the fact that the primary-key of $R_{m}$ is not affected by Remove; note that condition (1) of definition 4.2 is essential for satisfying this condition, and that if condition (2) of definition 4.2 is removed and the replacement of $Y_{i}$ with $K_{m}$ in inclusion dependencies of the form $R_{j}[Z] \subseteq R_{m}\left[Y_{i}\right]$ is allowed, then the third condition of definition 2.1 would not be satisfied.

\footnotetext{
Remove is applied on COURsE:" of figure 5 for:

O.C.NR, T.C.NR, and A.C.NR.

Relation-Scheme COURSE:" is replaced by COURSE" (C.NR, O.D.NAME, T.F.SSN, A.S.SSN)

Inclusion Dependencles involving COURSE:" are unchanged

Null Constraints involving COURSE" are replaced by :

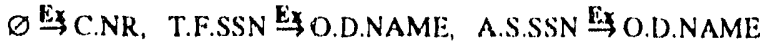

Fig. 6. Applying Remove on Relational Schema of Fig. 5.

\section{Applications of the Merging Technique}

In this section we discuss several aspects of applying our relation merging technique to relational databases that are implemented using a commercial relational database management system (DBMS), or to relational schemas developed using an EER-oriented design methodology.

\subsection{Relation Merging for Relational DBMSs}

The relation merging technique developed in section 4 involves merging relation-schemes into a new (merged) relation-scheme, and removing redundant attributes from the merged relation-scheme; a merged relation-scheme is associated with various null constraints, may be involved in non key-based inclusion dependencies (that are not referential integrity constraints), and may be associated with candidate keys that are allowed to be null. Some relational DBMSs do not have mechanisms for maintaining general null _onstraints, candidate keys that are allowed to be null, and non key-based inclusion dependencies; for such DBMSs our merging technique can be applied only when such constraints and dependencies are not generated.

Non key-based inclusion dependencies are not supported by DBMSs such as IBM's DB2 [5], but can be maintained in DBMSs such as SYBASE 4.0 [13] (using the triggers mechanism) and INGRES 6.3 [6] (using the rules mechanism). However, even in SYBASE and INGRES non key-based inclusion dependencies are harder to maintain then key-based inclusion dependencies. Keys that are allowed to be null cannot be maintained in DBMSs (e.g. SYBASE, INGRES) that consider all null values as identical. The conditions ensuring that Merge generates only key-based inclusion dependencies and keys consisting only of attributes that are not allowed io have null values, are given below (the type of inclusion and key dependencies is not affected by Remove ).

Proposition 5.1. Let $R S, R S^{\prime}, \vec{R}$, and Merge be defined as in definition 4.1, so that $R S^{\prime}=\left(R^{\prime}, F^{\prime} \cup I^{\prime} \cup N^{\prime}\right)$ is the result of applying Merge $(\bar{R})$ on $R_{s} S$, and $R_{m}\left(X_{m}\right)$ is the result of merging the relation-schemes of $\vec{R}$. Then (i) $I^{\prime}$ contains only key-based inclusion dependencies iff every relation-scheme $R_{i}\left(X_{i}\right)$ of $\bar{R}$ that is not a key-rciation, is not involved in the right-hand side of inclusion dependencies of the form $R_{j}[Z] \subseteq R_{i}\left[K_{i}\right], R_{j} \notin \bar{R}$; and (ii) the key attributes associated with $R_{m}$ are not allowed to have null values iff every relation-scheme $R_{i}\left(X_{i}\right)$ of $\bar{R}$ that is not a key-relation, is associated with a unique (primary) key.

Proof Sketch. The proof follows the specification of Merge .

Some DBMSs provide mechanisms for maintaining general null constraints. For example, the validproc mechanism of DB2, the triggers mechanism of SYBASE 4.0 , and the rules mechanisin of INGREs 6.3 can be used 
to maintain null constraints. However, these mechanisms require tedious and error-prone specifications of procedures, and therefore are difficult to use. Conversely, all relational DBMSs support declarative (non procedural) specifications for nulls-not-allowed constraints. The following proposition gives the conditions ensuring that the set of null constraints generated by Merge and simplified by Remove, consists only of nulls-not-allowed constraints.

Proposition 5.2. Let $R S, R S^{\prime}, \bar{R}$, and Merge be defined as in definition 4.1, so that $R S^{\prime}$ is the result of applying $\operatorname{Merge}(\bar{R})$ on $R S=(R, F \cup I \cup N)$, and $R_{m}\left(X_{m}\right)$ is the result of merging the relation-schemes of $\bar{R}$. Let Remove and $R S^{\prime \prime}$ be defined as in definition 4.3, so that the result of removing all removable attributes from $R_{m}\left(X_{m}\right)$ by applying Remove is $R S^{\prime \prime}=\left(R^{\prime \prime}, F^{\prime \prime} \cup I^{\prime \prime} \cup N^{\prime \prime}\right)$. Then $N^{\prime \prime}$ contains only nulls-not-allowed constraints if $\vec{R}$ contains a relation-scheme $R_{k}\left(X_{k}\right)$ such that for every relation-scheme $R_{i}\left(X_{i}\right)$ of $\vec{R}, R_{i} \neq R_{k}$, the following conditions are satisfied:

(1) $R_{i}\left[K_{i}\right] \subseteq R_{k}\left[K_{k}\right]$ belongs to $I$.

(2) $|Z|=1$, where $Z=X_{i}-K_{i}$, (i.e. $R_{i}$ has exactly one non primary-key attribute).

(3) $R_{i}$ is not involved in the right-hand side of any inclusion dependency of $I$.

(4) In addition to $R_{i}\left[K_{i}\right] \subseteq R_{k}\left[K_{k}\right], R_{i}$ can be involved only in the left-hand sides of inclusion dependencies of $I$ having the form $R_{i}[Z] \subseteq R_{j}\left[K_{j}\right]$ or $R_{i}\left[K_{i}\right] \subseteq R_{j}\left[K_{j}\right]$, where $R_{j} \notin \bar{R}$; however, if $R_{i}\left[K_{i}\right] \subseteq R_{j}\left[K_{j}\right]$ belongs to $I$ then $R_{k}\left[K_{k}\right] \subseteq R_{j}\left[K_{j}\right]$ also belongs to $I$.

Proof Sketch. Note that condition (1) implies that relation-scheme $R_{k}$ is a key-relation of $\vec{R}$. For part-null and total-equality constraints the proof follows from the definitions of Merge and Remove. Regarding nullsynchronization sets, nuli-existence constraints with empty right-hand sides are trivially satisfied. Finally, if all inclusion dependencies of $I$ involving relation-schemes of $\bar{R}$ in both their left-hand and right-hand sides, are of the form specified in (1) above, then Merge generates only nullexistence constraints that are either nulls-not-allowed constraints or belong to a null-synchronization set.

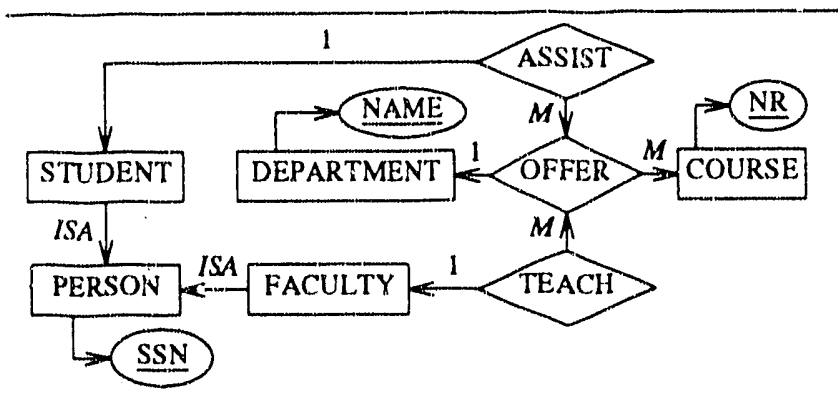

Fig. 7. An Extended Entity-Relationship Schema.

\subsection{Relation Merging for Relational Schema Translations of EER Schemas}

Relational schemas consisting of relation-schemes, key dependencies, key-based inclusion dependencies, and null constraints may represent EER schemas. The relational schema of figure 3 , for example, represents the EER schema of figure 7. Translations of EER schemas into relational schemas are discussed in detail in [11]. Note that if in relational schema translations of EER schemas every relation-scheme represents a single EER object-set, then the set of null constraints consists only of nulls-notallowed constraints involving primary-keys and foreignkeys [11] (e.g. see figure 3). These constraints express the usual restriction of not allowing EER entity-identifier attributes to have null values, and comply with the simplifying assumption in the definition of Merge .

ER and EER-oriented design methodologies for relational schemas recommend using a single relationscheme for representing a binary many-to-one relationship-set and the entity-set involved in that relationship-set with a many cardinality [14]. The result of applying the merging procedure developed in this paper on relational schema translations of EER schemas, shows that a single relation-scheme can be used for representing more complex structures as well (see figure 8). Such compact representations are especially useful for representing large generalization hierarchies whose specialization entity-sets are not involved in relationship-sets (see figure 8(i)). In most cases these compact representations require only additional null constraints. For example, in each of

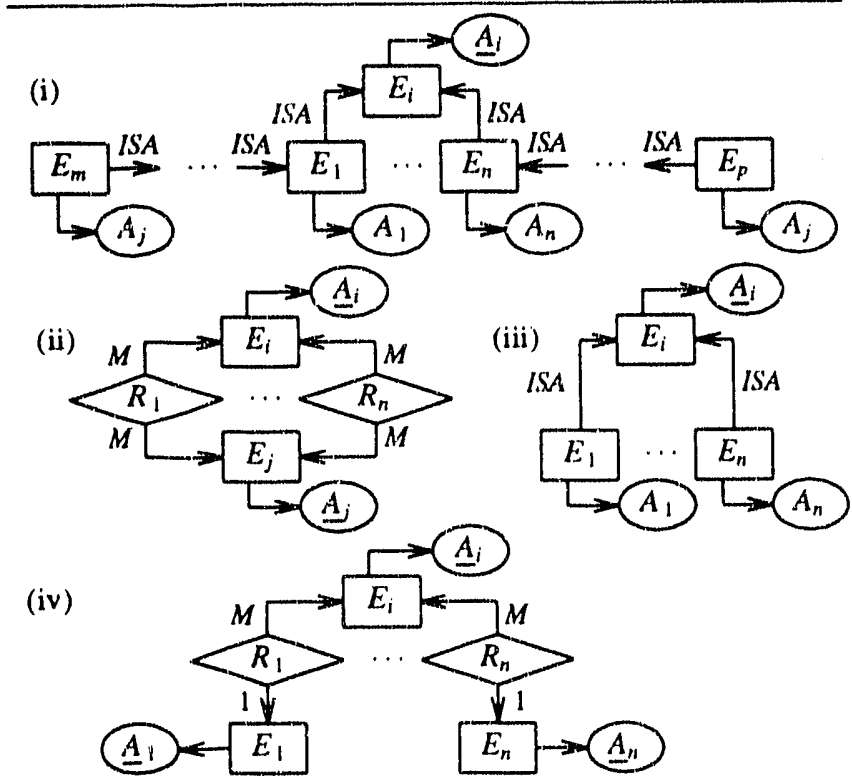

Fig. 8. EER Structures Amenable for Representations Involving a Single Relation. 
the EER structures shown in figure 8 multiple object-sets can be represented using a single relation-scheme; however, while for the EER schemas of figures 8(i) and 8(ii) this representation involves general null constraints, for the EER schemas of figures 8(iii) and 8(iv) this representation involves only nulls-not-allowed constraints. The conditions of proposition 5.2 imply that multiple object-sets can be represented by a single relation involving only nulls-not-allowed constraints, only if these multiple object-sets consist of:

(1) an entity-set $E_{i}$ and its specialization entity-sets, provided that these specialization entity-sets (a) have no specializations of theil own and are directly generalized only by $E_{i}$, (b) are not involved in relationshipsets or weak entity-sets, and (c) have exactly one (not inherited) attribute of their own (see figure 8(iii)); or

(2) an object-set $O_{i}$ and binar' many-to-one relationship-sets in which $O_{i}$ is involved with a many cardinality, provided that these relationship-sets (a) have no attributes, (b) are not involved in any other relationship-set, and (c) $O_{i}$ is associated by these relationship-sets with entity-sets that are not weak and have single-attribute identifiers (e.g. see figure 8(iv)).

Consider, for example, the EER schema of figure 7. Entity-set COURSE together with relationship-sets OFFER, TEACH, and ASSIST do not satisfy the conditions above, and, indeed, these object-sets can be represented using a single relation-scheme (such as relation-scheme COURSE" of figure 6) only if this relation-scheme is associated with null-existence constraints. Conversely, relationship-sets OFFER, TEACH, and ASSIST, satisfy conditions (2.a), (2.b), and (2.c), and therefore can "se represented using a single relation-scheme that is associated only with nulls-notallowed constraints.

\section{Summary}

We have preseisted in this paper a merging iechnique for relational schemas consisting of relation-schemes, key dependencies, referential integrity constraints, and null constraints. We have examined the conditions required for using this technique with relational DBMSs that provide different mechanisms for maintaining null and referential integrity constraints. For relational schemas developed using an EER-oriented design methodology, we have shown that a relation-scheme can be used for representing multiple object-sets not only for the standard binary many-to-one relationship-set structure, but for more complex structures as well.

Variations of the merging technique presented in this paper have been implemented as part of a database Schema Definition and Translation tool (SDT) [12]. Given an EER schema, SDT generates the corresponding schema definition for various relational DBMSs, such as DB2, SYBASE 4.0, and INGRES 6.3. SDT provides the options of (i) establishing a one-to-one correspondence between the relation-schemes in the relational schema and the object-sets in the EER schema (i.e. not using merging), or (ii) using merging for reducing the number of relationschemes in the relational schema.

\section{References}

[1] C. Beeri, P.A. Bernstein, and N. Goodman, "A sophisticate's introduction to database normalization theory", Proc. of the 4th VLDB Conference, 1978, pp. 113-124.

[2] P.P. Chen, "The entity-relationship model- towards a unified view of data", ACM Trans. on Database Systems 1,i (March 1976), pp. 9-36.

[3] E.P.F. Chan and P. Atzeni, "Independent database schemes under functional and inclusion dependencies", in Proc. of the 13th VLDB Conference, 1987. pp. 159-166.

[4] C.J. Date, "Referential integrity", in Relational Database-Selected Writings, Addison-Wesley, 1986.

[5] IBM Corporation, "IBM DATABASE 2 Referential Integrity Usage Guide", June 1989.

[6] Ingres, Inc., "INGRES/SQL Reference Manual", Release 6.3, Alameda, California, Nov. 1989.

[7] A. Klug, "Calculating Constraints on Relational Expressions", ACM Trans. on Database Systems 5,3 (Sep. 1980), pp. 260-290.

[8] R. Hull, "Relative information capacity of simple relational database schemata", in Proc. of the $3 \mathrm{rd}$ PODS Symposium, 1984, pp. 97-109.

[9] D. Maier, The theory of relational databases, C.omputer Science Press, 1983.

[10] D. Maier, D. Rozenshtein, and D.S. Warren, "Window functions", Advances in Computing Research. vol.3, JAI Press, 1986, pp. 213-246.

[11] V.M. Markowitz and A. Shoshani, "Representing extended entity-relationship structures in relational databases: A modular approach", to appear in $A C M$ Trans. on Database Systems.

[12] V.M. Markowitz and W. Fang, "SDT 4.1. Reference manual", 'TR LBL-27843, May 1991.

[13] Sybase, Inc., "Transact-SQL User's Guide", Release 4.0, Emeryville, California, Oct. 1989.

[14] T.J. Teorey, D. Yang, and J.P. Fry, "A logical design methodology for relational databases using the extended entity-relationship model", Computing Sur. veys 18,2 (June 1986), pp. 197-222. 

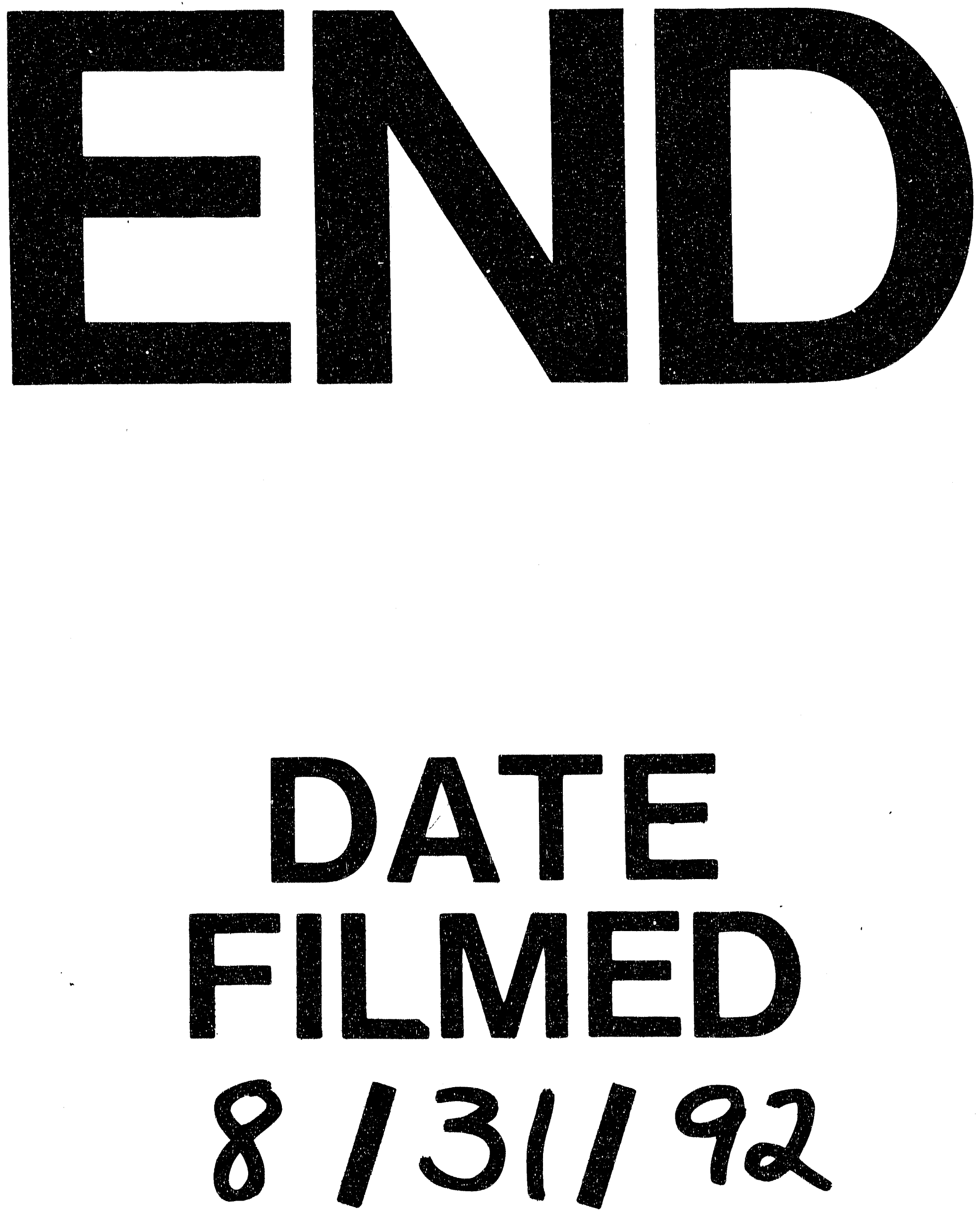